\title{
Unusual aspiration of foreign body in adults.
}

\author{
Reza Afghani ${ }^{*}$, Mahmoud Khandashpour Ghomi \\ ${ }^{1}$ Thoracic Surgeon, Department of Surgery, Azar Hospital, Golestan University of Medical Sciences, Gorgan, Iran \\ ${ }^{2}$ Pulmonologist, Bronchoscopy Ward, Sayad Shirazi Hospital, Golestan University of Medical Science, Gorgan, Iran
}

\section{Clinical Image}

Aspiration of foreign body can occur in children and adults. Children swallow objects due to their curiosity and are prone to aspiration. However in adults there are different risk factors for aspiration; such as alcohol consumption, addiction, drug consumption, senility, seizure, trauma, mental retardation, and artificial dentures [1]. In some cases, it may happen accidentally, and risk factors are not involved. In this particular case, air gun bullet has been swallowed (Figure 1). The shooter had put a bullet in his mouth in order to reload his gun and shoot rapidly; then swallowing and aspiration happened. In this case, because the foreign body is opaque, chest X-ray can be helpful for diagnosis (Figure 2). The alternative way to diagnose is fiberoptic bronchoscopy; which is sometimes considered a therapeutic strategy (Figure 3). Foreign body aspiration in tracheobronchial tree requires immediate diagnosis and removal; because the ignored objects may result in complications like infection, abscess, empyema, and haemoptysis or even bronchopleural fistula [2]. Therapeutic options to remove aspirated foreign bodies from the respiratory tract are carried out through fiberoptic and rigid bronchoscope [3]. In cases of immediate removal of the foreign body, the outcome will be excellent; and there will be no specific complication and sequel. In some rare cases, surgery is needed to remove the foreign body; however, surgery is mostly required in situations with late diagnosis and occurrence of complications.

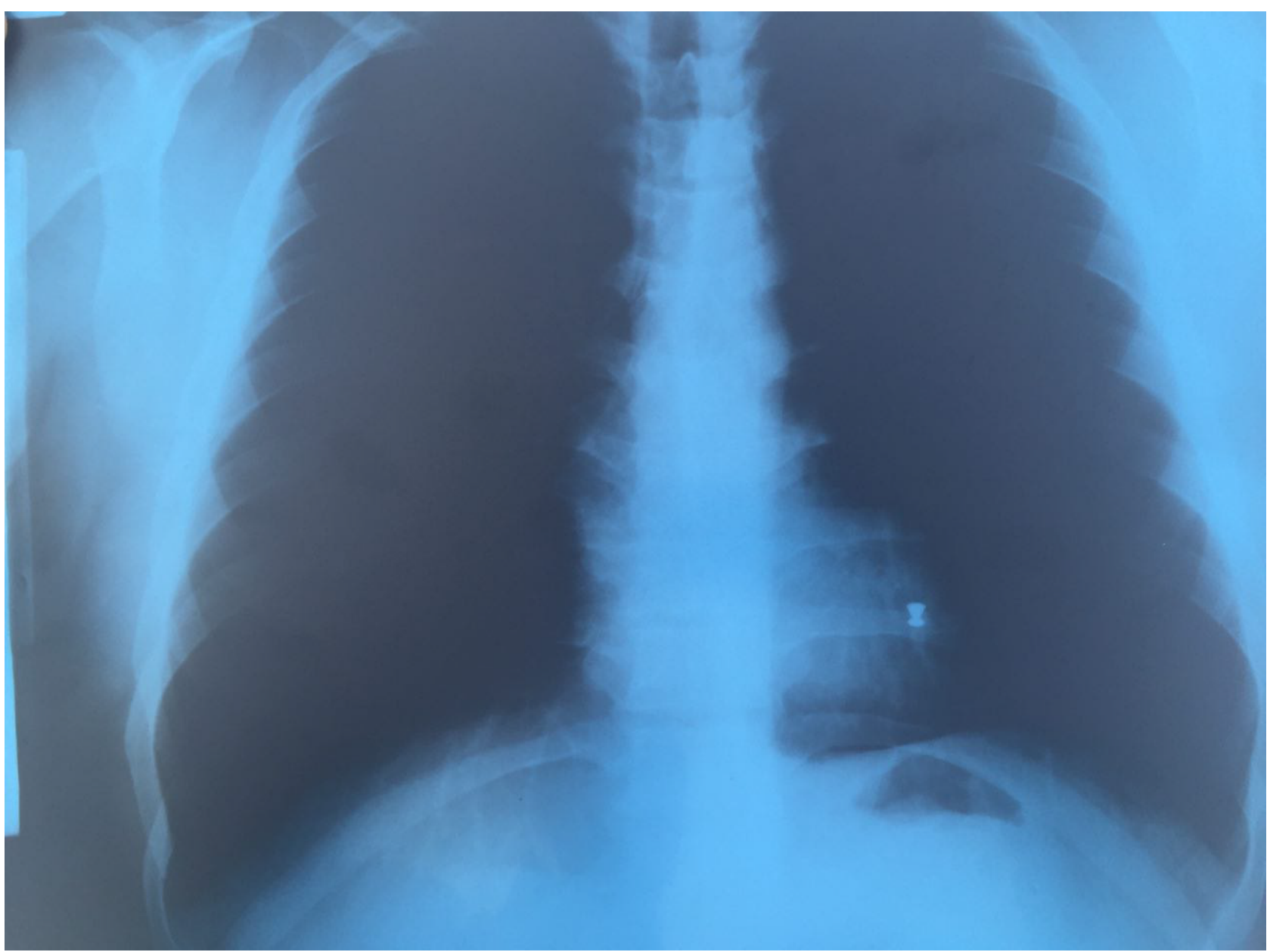

Figure 1. Foreign body. 


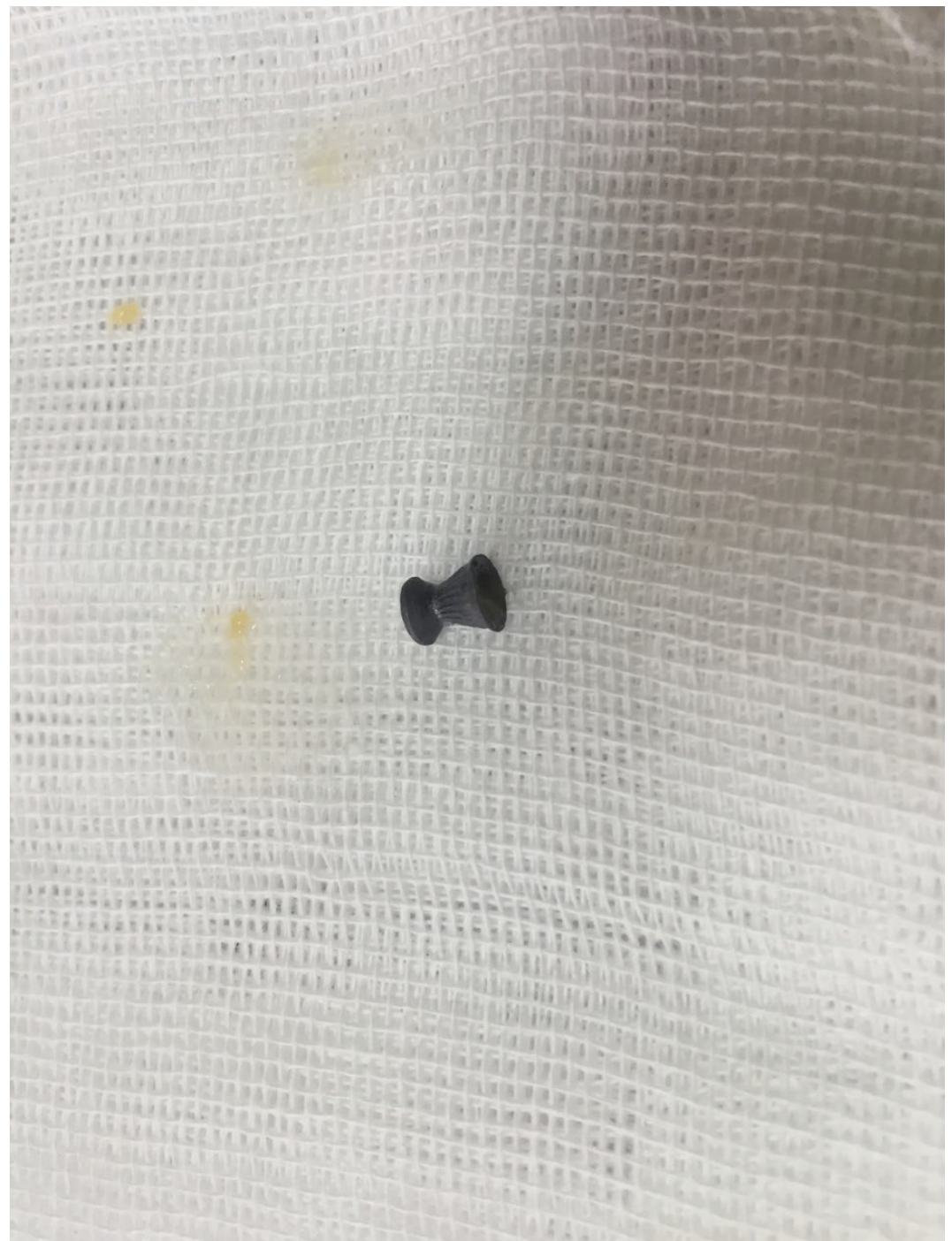

Figure 2. Chest X-ray of the patient.

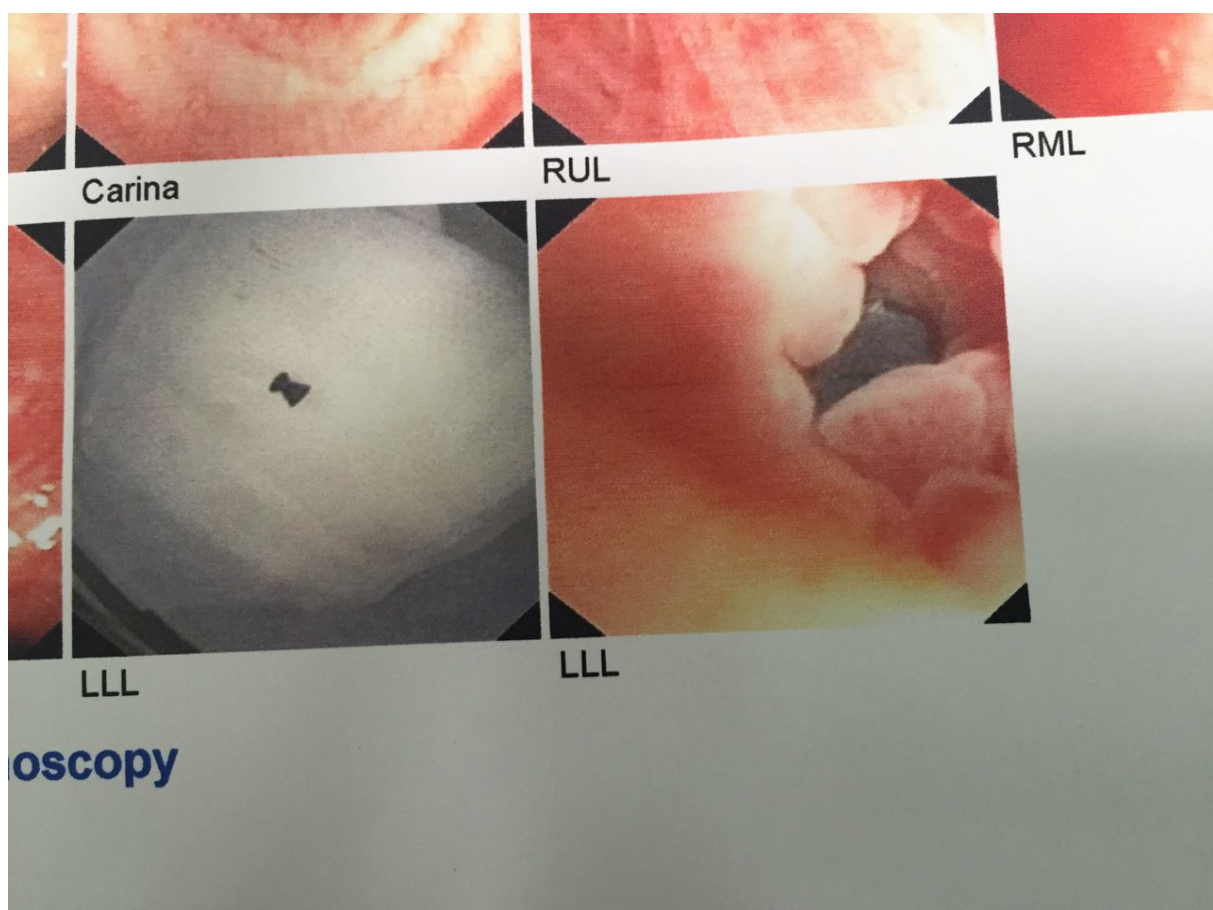

Figure 3. Bronchoscopic view and finding the foreign body in left lower lobe. 


\section{References}

1. Senturk E, Sen S. An unusual case of foreign body aspiration and review of the literature. Tuberk Toraks. 2011;59(2):173-7.

2. Bahnassy AA, Diab AB. Neglected bronchial foreign body in child simulating a calcified mass lesions:Challenging computed tomography diagnosis. Int $\mathrm{J}$ Health Sci. 2007;1(1):107-9.

3. Friedman EM. Tracheobronchial foreign bodies. Otolaryngol Clin North Am. 2000;33:179-85.

\section{*Correspondence to:}

Reza Afghani

Department of Surgery

Golestan University of Medical Sciences

Gorgan

Iran

Tel: +98 1732253500

E-mail: af_med75@yahoo.com 